\title{
Derived Subendocardial Viability Ratio and Cardiovascular Events in Patients with Chronic Kidney Disease
}

\author{
Robert Ekart $^{\text {a, b }}$ Sebastjan Bevc ${ }^{\text {b, } c}$ Nina Hojs ${ }^{b, c}$ Radovan Hojs ${ }^{\text {b, } c}$ \\ a Department of Dialysis, Clinic for Internal Medicine, University Medical Centre Maribor, \\ Maribor, Slovenia; ${ }^{b}$ Medical Faculty, University of Maribor, Maribor, Slovenia; ' Department \\ of Nephrology, Clinic for Internal Medicine, University Medical Centre Maribor, Maribor, \\ Slovenia
}

\section{Keywords}

Applanation tonometry · Cardiovascular mortality · Chronic kidney disease · Subendocardial viability ratio

\begin{abstract}
Background: Chronic kidney disease (CKD) is a well-known mortality risk factor. The subendocardial viability ratio (SEVR) is one of the pulse wave analysis parameters that constitutes a non-invasive measure of coronary perfusion. We aimed to assess the prognostic value of the SEVR for cardiovascular outcome in non-dialysis CKD patients. Methods: A total of 98 CKD patients (mean age 60 years) were prospectively followed up from the date of the SEVR measurement until their death or the start of dialysis/transplantation, maximally up to 7.1 years (mean 5 years). According to the manufacturer's instructions regarding normal SEVR values, the patients were divided into a low SEVR group (SEVR $\leq 130 \%, n=26)$ and a normal SEVR group (SEVR >130\%, $n=72$ ). Results: During the follow-up period, 13 patients (13.3\%) suffered fatal and 23 patients (23.5\%) suffered combined (non-fatal and fatal) cardiovascular events. In the patients who died of cardiovascular causes, the SEVR values were statistically significantly lower (130 vs. $154 \% ; p=0.017$ ) than in those who survived. A Kaplan-Meier survival analysis showed that the cardiovascular survival rate in the low SEVR group of patients was statistically significantly lower (log-rank test: $p<0.001$ ). Using an unadjusted Cox regression analysis, the patients in the low SEVR group had a 5.6-fold higher risk $(95 \% \mathrm{Cl}$ : $1.8-17.3$; $p=0.002$ ) of fatal cardiovascular events and a 2.7 -fold higher risk ( $95 \% \mathrm{Cl}: 1.1-6.3 ; p=0.024$ ) of combined fatal and non-fatal cardiovascular events. In the adjusted Cox regression model, the patients in the low SEVR group had a 16 -fold higher risk $(95 \% \mathrm{Cl}: 1.2-9.7 ; p=0.004)$ of fatal cardiovascular events and a 7 -fold higher risk (95\% Cl: $1-9.7 ; p=0.009)$ of combined fatal and non-fatal cardiovascular events. Conclusions: An SEVR $<130 \%$ predicts fatal and nonfatal cardiovascular events in non-dialysis CKD patients.


Ekart et al.: Derived SEVR and CV Events in CKD Patients

\section{Introduction}

Chronic kidney disease (CKD) is an established independent risk factor for increased cardiovascular events and cardiovascular mortality [1]. Among CKD patients, the risk of cardiovascular death is much higher than the risk of reaching an end-stage kidney disease and dialysis treatment. CKD patients are 10-20 times more likely to die of cardiovascular diseases than the general population, and dialysis-dependent patients are at an even higher risk [2]. Central haemodynamic indices and pulse wave analysis (PWA) are listed in the ESH/ESC criteria for cardiovascular risk assessment [3]. The subendocardial viability ratio (SEVR) is one of the measured PWA parameters and can be assessed non-invasively by applanation tonometry. The SEVR is related to the work of the heart and oxygen consumption, as well as the energy supply of the heart $[4,5]$. Low SEVR values indicate reduced subendocardial perfusion [5-7]. A low SEVR has been associated with albuminuria [8-10] and haemoglobin [11], as well as with markers of inflammation in patients with rheumatoid arthritis [12]. Hypertensive patients with reduced coronary flow reserve [13], extreme dippers [14], patients with rheumatoid arthritis [15] or peripheral artery disease [16], women with type 2 diabetes [17] and highly proteinuric CKD patients with an estimated glomerular filtration rate (eGFR) $<30 \mathrm{~mL} / \mathrm{min} / 1.73 \mathrm{~m}^{2}$ [18] have also been associated with a lower SEVR. Recently, a reduced SEVR was shown to predict cardiovascular mortality in CKD patients [19] and combined endpoint all-cause mortality and end-stage renal disease in patients with type 1 diabetes [10].

Studies about the association between the SEVR and long-term outcome in CKD patients are sparse. We aimed to investigate the association between the SEVR as a prognostic factor and non-fatal, fatal and combined cardiovascular events in a cohort of non-dialysis CKD patients.

\section{Subjects and Methods}

\section{Study Participants}

Patients were recruited between October 2010 and November 2017 from the outpatient nephrology clinic at the University Medical Centre Maribor, Slovenia. Inclusion criteria were an age $>18$ years and a diagnosis of CKD (markers of kidney damage and/or a decreased eGFR) according to the Kidney Disease: Improving Global Outcomes (KDIGO) definition and classification of CKD [20]. Exclusion criteria at the time of enrolment of the patients in the study were atrial fibrillation, aortic valve stenosis, acute coronary syndrome, acute heart failure, acute infectious disease and/or the need for systemic antibiotic therapy, active cancer disease, acute renal failure, renal replacement therapy and pregnancy.

Pulse Wave Analysis

All patients were examined between 8 a.m. and 11 a.m. A single examiner performed all measurements. Before measurement, the subjects had been in a fasting state for $<12 \mathrm{~h}$ and abstained from cigarettes, coffee, tea and exercise. Before the PWA measurement, and after a minimum of $10 \mathrm{~min}$ of rest, office brachial diastolic and systolic blood pressure values were taken from the portable bedside automatic blood pressure monitoring device (Dash 4000; General Electric Healthcare, Dallas, TX, USA). During the whole procedure, the patients were seated comfortably beside a table with their arm resting on the table and their palm facing upward. Radial artery pressure waveforms were recorded at least 2 times using applanation tonometry (SphygmoCor ${ }^{\circledR}$; AtCor Medical, Ltd., Sydney, NSW, Australia). The SphygmoCor ${ }^{\circledR}$ Central Blood Pressure Assessment System uses a high-fidelity Millar pressure transducer (Millar Instruments, Houston, TX, USA) to non-invasively record the pressure wave at the radial artery. This is achieved by partially flattening the radial artery against the underlying bone in the wrist. The key measurements of cardiovascular risk and heart function are calculated from this aortic pressure waveform. According to its classic definition, the SEVR is a pressure-time integral ratio derived from the pressures measured in the aorta and the left ventricle [5, 21]. The SphygmoCor ${ }^{\circledR}$ Software calculates the SEVR as the ratio of the area under the diastolic part of the derived 
Ekart et al.: Derived SEVR and CV Events in CKD Patients

Fig. 1. Subendocardial viability

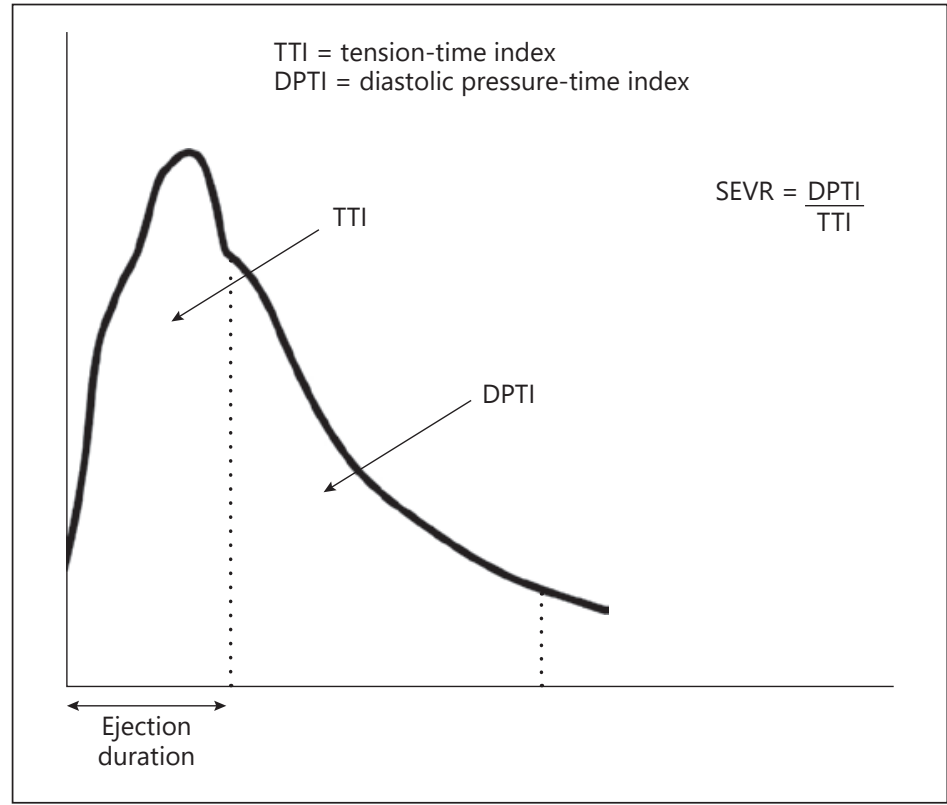
ratio (SEVR).

aortic pressure waveform (the diastolic pressure-time index [DPTI]) to the area under the systolic section of the waveform (the tension-time index [TTI]), i.e., DPTI/TTI (Fig. 1). The SEVR for normal conditions is usually high (130-200\%) [4].

Laboratory Variables

At baseline, fasting blood samples were drawn under standardised conditions. The eGFR was calculated via the Chronic Kidney Disease Epidemiology Collaboration (CKD-EPI) creatinine-cystatin C formula [22].

Outcome

All patients were followed up in order to record episodes of non-fatal or fatal cardiovascular events up until 20 December 2017. A non-fatal event was defined as the occurrence of non-fatal acute coronary syndrome (unstable angina pectoris, non-transmural or transmural myocardial infarction), heart failure with hospitalisation, stroke (ischaemic or haemorrhagic) or peripheral arterial disease (gangrene, bypass surgery or percutaneous transluminal angioplasty). A fatal event was death caused by myocardial infarction, sudden death, heart failure or stroke. Some patients had more than one non-fatal event, some non-fatal events and a fatal event, and others only a fatal event. All events were verified by the medical records in the hospital's medical computer program.

Patients who started with dialysis $(n=26)$ or underwent pre-emptive renal transplantation $(n=6)$ were censored at the time they started dialysis or transplantation, because the cardiovascular risk for these two groups is different than for pre-dialysis patients.

\section{Statistical Analysis}

The outcome events studied were non-fatal, fatal and combined cardiovascular events. The numbers of patients with these events were analysed. Normally, distributed variables are presented as mean values and range or standard deviation (SD), and compared with Student $t$ tests, while non-normally distributed variables are expressed as median (interquartile range [IQR]) and compared using Mann-Whitney U tests. Interobserver variability in SEVR measurements was tested using an intraclass correlation coefficient (ICC), which reflects agreement between measurements. ICC estimates and their 95\% confidence intervals (CIs) were calculated based on a mean-rating $(\mathrm{k}=2)$, absolute-agreement, two-way mixed-effects model. Correlation (by Pearson coefficient) between different variables was performed.

According to the manufacturer's instructions regarding normal SEVR values, the patients were divided into a low SEVR group (SEVR $\leq 130 \%$ ) and a normal SEVR group (SEVR >130\%). Fisher's exact test was used to compare differences between two independent groups when the dependent variable was ordinal. The 
Ekart et al.: Derived SEVR and CV Events in CKD Patients
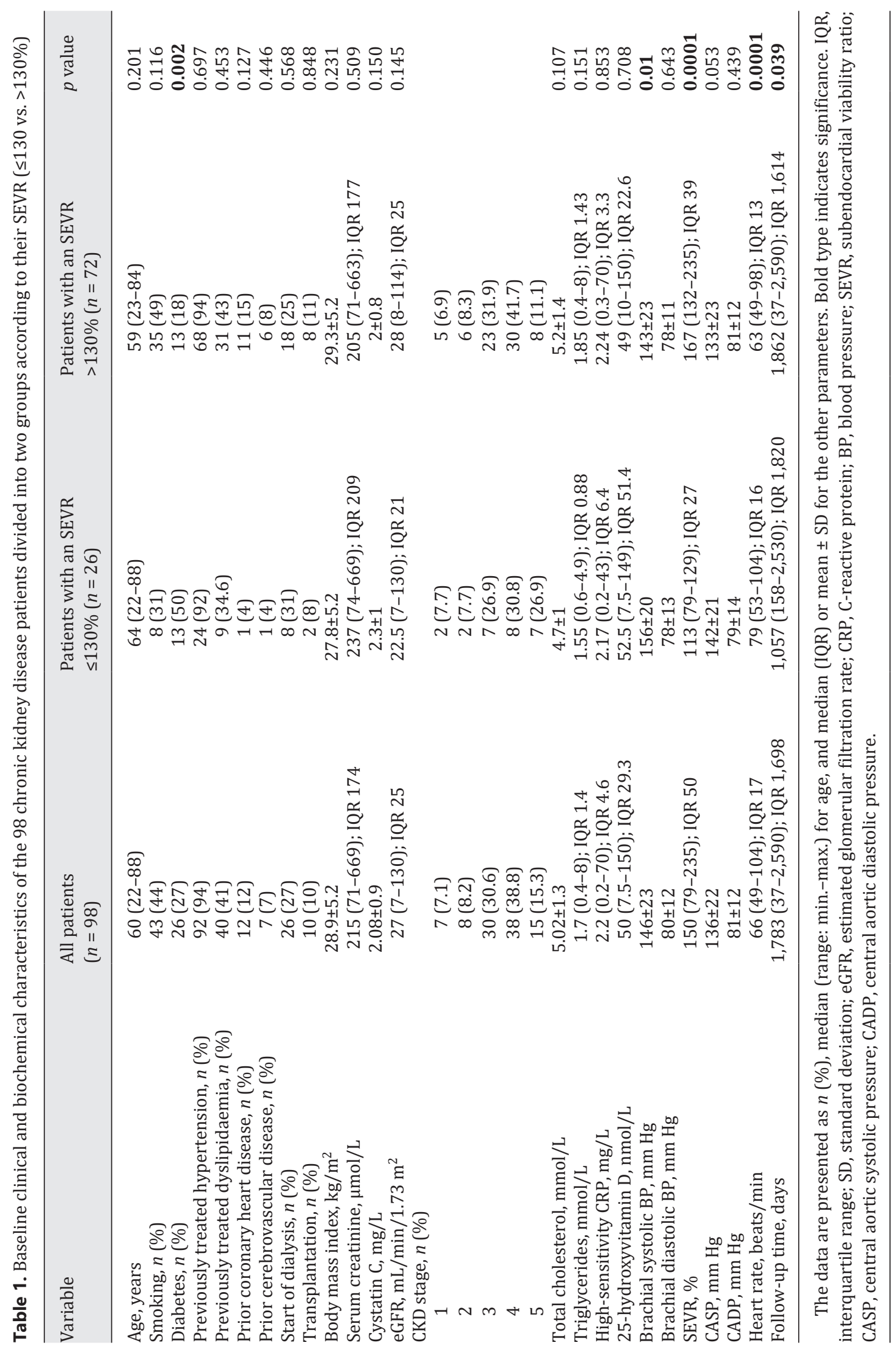
Ekart et al.: Derived SEVR and CV Events in CKD Patients

Table 2. Patients with cardiovascular events among those with an SEVR $\leq 130 \%$ and those with an SEVR $>130 \%$

\begin{tabular}{llll}
\hline & SEVR $\leq 130 \%$ & SEVR $>130 \%$ & $p$ value \\
\hline Patients & 26 & 72 & - \\
Non-fatal cardiovascular events & $3(11.5)$ & $11(15.3)$ & 0.754 \\
$\quad$ Acute coronary syndrome & $1(3.8)$ & $8(11.1)$ & 0.438 \\
$\quad$ Heart failure & $1(3.8)$ & $3(4.2)$ & - \\
Stroke & 0 & $1(1.4)$ & - \\
$\quad$ Ischaemic stroke & 0 & $1(1.4)$ & - \\
$\quad$ Haemorrhagic stroke & 0 & 0 & - \\
Peripheral artery disease & $2(7.7)$ & $4(5.6)$ & 0.654 \\
Fatal cardiovascular events & $8(30.8)$ & $5(6.9)$ & $\mathbf{0 . 0 0 5}$ \\
$\quad$ Myocardial infarction & 0 & $1(1.4)$ & - \\
$\quad$ Sudden death & $3(11.5)$ & $2(2.8)$ & - \\
$\quad$ Heart failure & $4(15.4)$ & $2(2.8)$ & - \\
$\quad$ Stroke & $1(3.8)$ & 0 & - \\
Combined cardiovascular events & $9(34.6)$ & $14(19.4)$ & 0.175 \\
\hline
\end{tabular}

Data are presented as $n$ or $n(\%)$. Bold type denotes significance. SEVR, subendocardial viability ratio.

relationship between the SEVR and cardiovascular events was tested by stratifying the patients into two groups according to their SEVR values being $\leq 130$ or $>130 \%$.

Survival rates were estimated using Kaplan-Meier survival curves and compared by the Mantel (logrank) test. A multivariate Cox regression model was used to assess the influence of traditional cardiovascular risk variables (age, diabetes, smoking, cholesterol, brachial systolic blood pressure and eGFR) on cardiovascular events. A value of $p<0.05$ was considered statistically significant. All of the statistical analyses were performed with the Statistical Package for the Social Sciences for Windows version 25.0 (SPSS Inc., Chicago, IL, USA).

\section{Results}

\section{Baseline Characteristics}

The mean age of the 98 patients at the time of enrolment in the study was 60 years, and $67 \%$ were men. The most frequent causes of CKD were hypertensive nephropathy (41\%), diabetic nephropathy $(24.5 \%)$, chronic glomerulonephritis $(15 \%)$ and polycystic kidney disease (7\%). Twenty-six patients (26.5\%) were in the low SEVR group and 72 patients (73.5\%) in the normal SEVR group. The descriptive (demographic, clinical and biochemical) data on the patients studied and the comparison of variable values between the two groups of patients according to SEVRs $\leq 130$ or $>130 \%$ are presented in Table 1 . The ICC for SEVR measurements was 0.98 with a $95 \% \mathrm{CI}$ between 0.98 and 0.99 . We found a statistically significant correlation between SEVR and heart rate $(r=-0.624 ; p=0.0001)$. The patients in the low SEVR group had statistically significantly higher heart rates $(p=0.0001)$.

In Table 2, patients with different cardiovascular events are presented by SEVR group. One patient suffered 2 non-transmural myocardial infarctions, 1 patient had 5 and 1 patient had 4 hospitalisations due to heart failure, and 1 patient had 4 hospitalisations due to peripheral arterial disease. None of these 4 patients had an SEVR $<130 \%$. After the initial assessment, the patients were followed up for 37-2,590 days (median 1,738; IQR 1,698). 


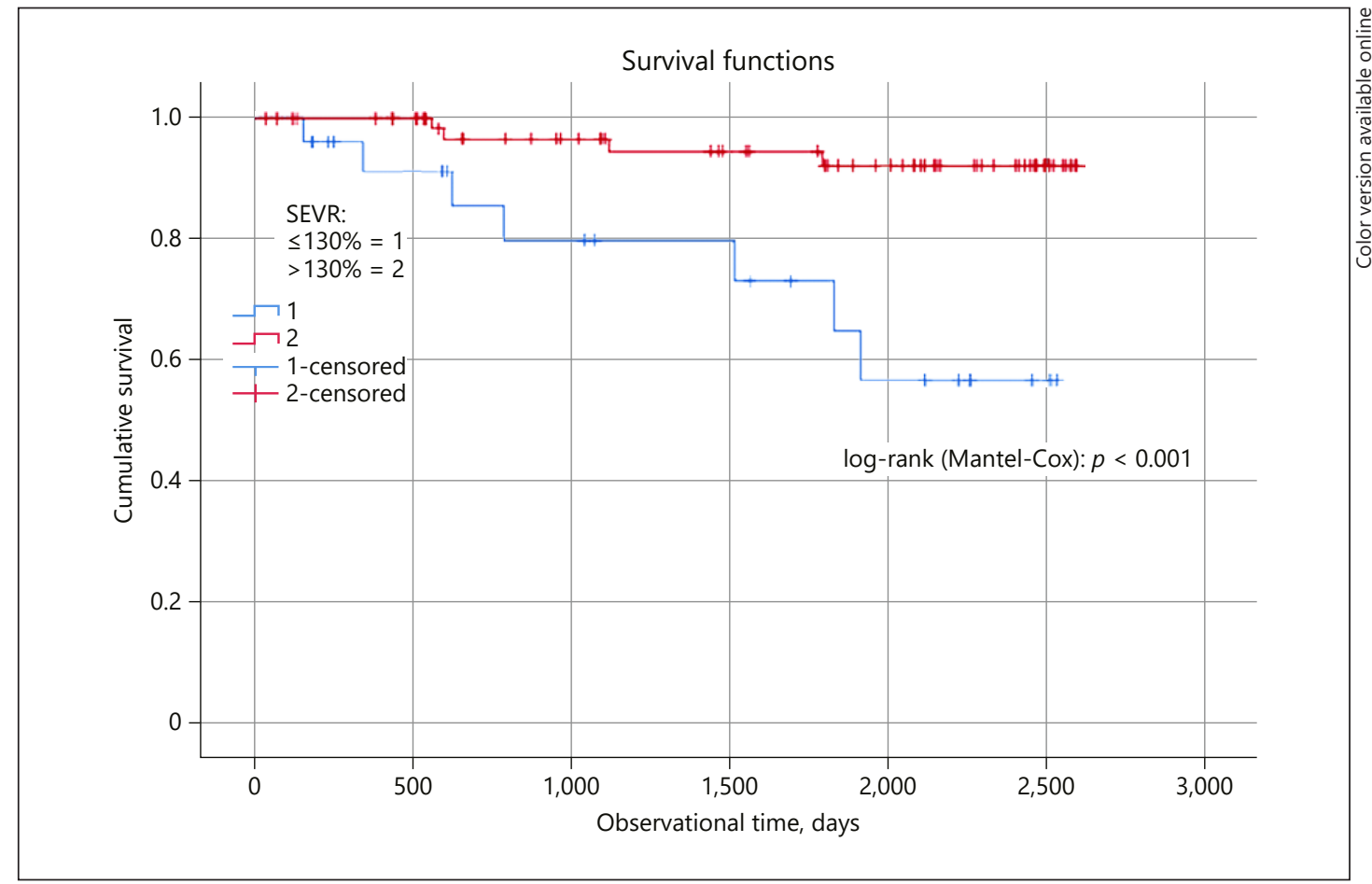

Fig. 2. Kaplan-Meier survival curves for the rate of fatal cardiovascular events, stratified by subendocardial viability ratio (SEVR) ( $\leq 130$ vs. >130\%). Observation period: from October 2010 to 20 December 2017 or start of dialysis/transplantation or date of death.

Prognostic Value of the SEVR for Non-Fatal Cardiovascular Events

During the follow-up period, 14 patients (14.3\%) suffered a non-fatal cardiovascular event. The results of the Kaplan-Meier survival analysis did not reveal any difference between the groups with low and normal SEVRs (log-rank [Mantel-Cox]: $p=0.698$ ). In the unadjusted Cox regression model, a low SEVR was not an independent predictor of non-fatal cardiovascular events $(p=0.699)$.

\section{Prognostic Value of the SEVR for Fatal Cardiovascular Events}

During follow-up, 13 patients (13.3\% of all enrolled patients) died from a fatal cardiovascular event. The results of the Kaplan-Meier survival analysis showed that the survival rate in the low SEVR group $(\leq 130 \%)$ was significantly lower than in the normal SEVR group $(>130 \%)$ (log-rank test: $p<0.001)$, with a mean $( \pm$ SD) survival of 1,222 $( \pm 865)$ days and $1,622( \pm 825)$ days, respectively (Fig. 2). In the univariable Cox regression analysis, the hazard ratio for a fatal cardiovascular event in the low SEVR group was 5.646 (95\% CI: 1.841-17.311; $p=0.002$ ). In the multivariable Cox regression model, which included age, diabetes, smoking, cholesterol, systolic blood pressure, heart rate and eGFR, only heart rate $(p=0.038)$ and SEVR $<130 \%(p=0.004)$ turned out to be independent predictors of a fatal cardiovascular event (Table 3).

Prognostic Value of the SEVR for Combined Non-Fatal and Fatal Cardiovascular Events

During follow-up, 23 patients (23.5\%) suffered a combined (non-fatal and fatal) cardiovascular event; 9 patients were in the low SEVR group and 14 patients in the normal SEVR group ( $p=0.118$ ) (Table 2). The results of the Kaplan-Meier survival analysis revealed that 
Ekart et al.: Derived SEVR and CV Events in CKD Patients

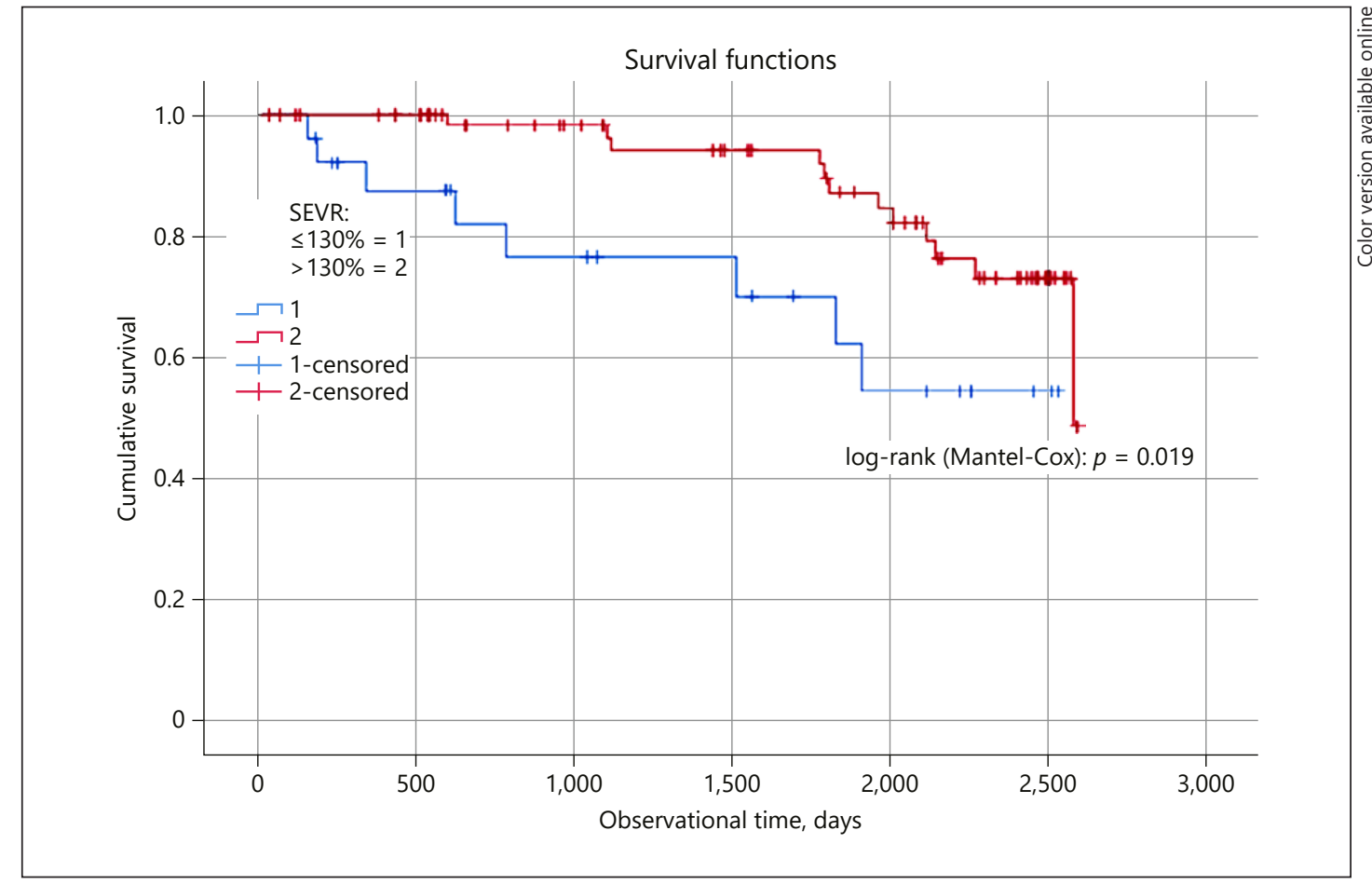

Fig. 3. Kaplan-Meier survival curves for combined fatal and non-fatal cardiovascular events, stratified by subendocardial viability ratio (SEVR) ( $\leq 130$ vs. $>130 \%$ ). Observation period: from October 2010 to 20 December 2017 or start of dialysis/transplantation or date of death.

Table 3. Multivariable Cox proportional-hazards regression analysis for prediction of non-fatal, fatal and combined (non-fatal and fatal) cardiovascular events

\begin{tabular}{llll}
\hline Variable & $\begin{array}{l}\text { Non-fatal } \\
\text { cardiovascular events }\end{array}$ & $\begin{array}{l}\text { Fatal } \\
\text { cardiovascular events }\end{array}$ & $\begin{array}{l}\text { Combined } \\
\text { cardiovascular events }\end{array}$ \\
\hline Age & $0.992 ; 0.935-1.055$ & $0.976 ; 0.907-1.05$ & $0.992 ; 0.943-1.043$ \\
Diabetes & $1.864 ; 0.46-7.545$ & $3.451 ; 0.986-12.074$ & $2.299 ; 0.836-6.342$ \\
Smoking & $3.531 ; 0.882-14.139$ & $2.347 ; 0.575-9.58$ & $2.946 ; 1.059-8.196^{*}$ \\
Cholesterol & $0.415 ; 0.199-0.864^{*}$ & $0.698 ; 0.369-1.322$ & $0.684 ; 0.414-1.132$ \\
eGFR & $0.975 ; 0.944-1.007$ & $0.967 ; 0.929-1.007$ & $0.968 ; 0.943-0.994^{*}$ \\
Systolic blood pressure & $1.044 ; 1.017-1.072^{*}$ & $1.022 ; 0.987-1.057$ & $1.04 ; 1.019-1.062^{*}$ \\
Heart rate & $0.977 ; 0.901-1.059$ & $0.919 ; 0.849-0.995^{*}$ & $0.946 ; 0.892-1.004$ \\
SEVR $\leq 130 \%$ & $5.179 ; 0.438-61.214$ & $16.171 ; 2.42-108.039^{*}$ & $7.472 ; 1.659-33.651^{*}$ \\
\hline
\end{tabular}

Values denote "risk ratio; 95\% CI." * $p<0.05$. SEVR, subendocardial viability ratio; eGFR, estimated glomerular filtration rate.

the survival rate in the low SEVR group $(\leq 130 \%)$ was statistically significantly lower than in the normal SEVR group (>130\%) (log-rank test: $p<0.019)$ (Fig. 3). In the unadjusted Cox regression analysis, the hazard ratio for a combined cardiovascular event in the low SEVR group was 2.679 (95\% CI: 1.139-6.302; $p=0.024$ ). In the multivariable Cox regression model, which included age, diabetes, smoking, cholesterol, systolic blood pressure, heart rate, eGFR 
and SEVR, only smoking ( $p=0.038)$, eGFR $(p=0.016)$, systolic blood pressure $(p=0.0001)$ and SEVR $\leq 130 \%(p=0.009)$ turned out to be independent predictors of a combined cardiovascular event (Table 3).

\section{Discussion}

We evaluated the prognostic value of SEVR measurements in non-dialysis CKD patients. The main result of this study is the association between SEVR values $\leq 130 \%$ and a higher rate of fatal and combined cardiovascular events. We did not find an association between SEVR values $\leq 130 \%$ and non-fatal cardiovascular events. The SEVR represented a new independent predictor of cardiovascular mortality in these patients. According to our results, the SEVR may be usefully applied for mortality risk stratification in the CKD population.

According to our knowledge, this study had the longest follow-up (up to 7 years, mean 5 years). There was one prior post hoc analysis study on stage 3-4 CKD patients relating the SEVR to survival, in which the follow-up duration was 36 months from the study's beginning [19]. Therefore, the two studies are complementary and, together, strongly suggest that reduced SEVR values could have detrimental consequences on CKD patients. Our study used the longer follow-up, and stage 1-5 CKD patients were analysed.

Peripheral applanation tonometry-based measurement of the SEVR should be considered to be a sensitive, reproducible and validated measure of the balance between the supply with and the demand for myocardial oxygen, as well as of the adequacy of subendocardial perfusion [13]. It is very well known that CKD patients are prone to cardiovascular disease and that many of them have left ventricular hypertrophy, coronary heart disease and heart failure. CKD is established as an independent risk factor for cardiovascular disease. The patients included in this study had no symptoms of acute cardiovascular disease. The group of patients with an SEVR $\leq 130 \%$ died earlier, most of them of cardiovascular disease. In previous studies, associations between a low SEVR and many unfavourable factors have been found [8-19]. Our study showed that patients with a lower SEVR had a 16-fold higher risk of cardiovascular death and a 7-fold higher risk of combined cardiovascular events. Half of the patients with a lower SEVR had diabetes, whereas there were only $18 \%$ diabetics in the group with normal SEVR. However, the difference was not statistically significant.

The pathophysiologic explanation for our results is that patients with a lower SEVR have a shorter DPTI and a higher heart rate than patients with a higher SEVR. Heart rate and SEVR were also statistically significant predictors of fatal cardiovascular events. Since the left ventricular subendocardial flow is mainly or entirely diastolic, it should depend on coronary driving pressure and duration of diastole [5]. In a study by Buckberg et al. [5], diastolic duration correlated better with the distribution of blood flow than it did with any other variable. Any reduction in diastolic time would lower the DPTI further and could jeopardise the subendocardial flow [5]. For this reason, a reduction in SEVR corresponds to an impaired subendocardial flow [23]. According to these explanations, we can hypothesise that patients with a low SEVR - despite having no symptoms of coronary heart disease - are, together with a higher heart rate, predisposed to develop myocardial ischaemia and further cardiovascular complications, which results in a higher rate of cardiovascular mortality.

The major limitation of this study is its small sample size. Caution should be used in interpreting the results of the Cox regression analysis, due to the number of covariates and the small number of events. Another limitation of our study is that we did not perform echocardiography at baseline. Also, we did not conduct a controlled SEVR measurement, which might have answered whether SEVR values were changing or diminishing over time. Further 
Ekart et al.: Derived SEVR and CV Events in CKD Patients

randomised and interventional studies are needed to test the influence of different risk factors on the SEVR and cardiovascular mortality.

In conclusion, our study showed that SEVR values $\leq 130 \%$ have prognostic value for the prediction of fatal and non-fatal cardiovascular events in non-dialysis CKD patients.

\section{Statement of Ethics}

The study was approved by the National Ethics Committee and conducted in concordance with the Helsinki Declaration; all participants signed their written informed consent.

\section{Disclosure Statement}

The authors declare that there is no conflict of interest.

\section{References}

1 Sarafidis PA, Bakris GL. Cardiovascular disease in CKD in 2014: new insights into cardiovascular risk factors and outcomes. Nat Rev Nephrol. 2015;11(2):70-2.

2 Gregg LP, Hedayati SS: Management of Traditional Cardiovascular Risk Factors in CKD: What Are the Data? Am J Kidney Dis. 2018 [Epub ahead of print].

3 Vlachopoulos C, Xaplanteris P, Aboyans V, Brodmann M, Cífková R, Cosentino F, et al. The role of vascular biomarkers for primary and secondary prevention. A position paper from the European Society of Cardiology Working Group on peripheral circulation: Endorsed by the Association for Research into Arterial Structure and Physiology (ARTERY) Society. Atherosclerosis. 2015;241(2):507-32.

4 SphygmoCor: A Clinical Guide, Pulse Wave Analysis [Internet]. Available from: https://www.ttu.ee/public/t/ Tehnomeedikum/Instituudid/Biomeditsiinitehnika_instituut/Oppetoo/Oppematerjalid/Sphygmocor_-Clinical_Guide.pdf

5 Buckberg GD, Fixler DE, Archie JP, Hoffman JI. Experimental subendocardial ischemia in dogs with normal coronary arteries. Circ Res. 1972;30(1):67-81.

6 Sarnoff SJ, Braunwald E, Welch GH Jr, Case RB, Stainsby WN, Macruz R. Hemodynamic determinants of oxygen consumption of the heart with special reference to the tension-time index. Am J Physiol. 1958;192(1):148-56.

7 Buckberg GD, Towers B, Paglia DE, Mulder DG, Maloney JV. Subendocardial ischemia after cardiopulmonary bypass. J Thorac Cardiovasc Surg. 1972;64:669-84.

8 Ekart R, Bevc S, Hojs N, Knehtl M, Dvoršak B, Hojs R. Albuminuria is Associated with subendocardial viability ratio in chronic kidney disease patients. Kidney Blood Press Res. 2015;40(6):565-74.

9 Prince CT, Secrest AM, Mackey RH, Arena VC, Kingsley LA, Orchard TJ. Augmentation pressure and subendocardial viability ratio are associated with microalbuminuria and with poor renal function in type 1 diabetes. Diab Vasc Dis Res. 2010;7(3):216-24.

10 Theilade S, Hansen TW, Rossing P. Central hemodynamics are associated with cardiovascular disease and albuminuria in type 1 diabetes. Am J Hypertens. 2014;27(9):1152-9.

11 Ekart R, Bevc S, Hojs N, Stropnik Galuf T, Hren M, Dvoršak B, et al. Relationship between subendocardial viability ratio and hemoglobin in patients with chronic kidney disease. Clin Nephrol. 2017 Supplement 1; 88(13):22-6.

12 Sandoo A, Protogerou AD, Hodson J, Smith JP, Zampeli E, Sfikakis PP, et al. The role of inflammation, the autonomic nervous system and classical cardiovascular disease risk factors on subendocardial viability ratio in patients with RA: a cross-sectional and longitudinal study. Arthritis Res Ther. 2012 Nov;14(6):R258.

13 Tsiachris D, Tsioufis C, Syrseloudis D, Roussos D, Tatsis I, Dimitriadis K, et al. Subendocardial viability ratio as an index of impaired coronary flow reserve in hypertensives without significant coronary artery stenoses. J Hum Hypertens. 2012;26(1):64-70.

14 Amah G, Ouardani R, Pasteur-Rousseau A, Voicu S, Safar ME, Kubis N, et al. Extreme-Dipper Profile, Increased Aortic Stiffness, and Impaired Subendocardial Viability in Hypertension. Am J Hypertens. 2017 Apr;30(4): 417-26.

15 Anyfanti P, Triantafyllou A, Gkaliagkousi E, Triantafyllou G, Koletsos N, Chatzimichailidou S, et al. Subendocardial viability ratio in patients with rheumatoid arthritis: comparison with healthy controls and identification of prognostic factors. Clin Rheumatol. 2017;36(6):1229-36.

16 Mosimann K, Jacomella V, Thalhammer C, Meier TO, Kohler M, Amann-Vesti B, et al. Severity of peripheral arterial disease is associated with aortic pressure augmentation and subendocardial viability ratio. J Clin Hypertens (Greenwich). 2012;14(12):855-60. 
17 Laugesen E, Høyem P, Fleischer J, Kumarathas I, Knudsen ST, Hansen KW, et al. Reduced Subendocardial Viability Ratio Is Associated With Unfavorable Cardiovascular Risk Profile in Women With Short Duration of Type 2 Diabetes. Am J Hypertens. 2016;29(10):1165-72.

18 Ekart R, Šegula A, Hartman T, Hojs N, Hojs R. Subendocardial Viability Ratio Is Impaired in Highly Proteinuric Chronic Kidney Disease Patients With Low Estimated Glomerular Filtration Rate. Ther Apher Dial. 2016; 20(3):281-5.

19 Di Micco L, Salvi P, Bellasi A, Sirico ML, Di Iorio B. Subendocardial viability ratio predicts cardiovascular mortality in chronic kidney disease patients. Blood Purif. 2013;36(1):26-8.

20 Kidney Disease: Improving Global Outcomes (KDIGO) CKD Work Group. KDIGO 2012 Clinical Practice Guideline for the Evaluation and Management of Chronic Kidney Disease. Kidney Int Suppl. 2013;3:1-150.

21 Hoffman JI, Buckberg GD. The myocardial supply:demand ratio - a critical review. Am J Cardiol. 1978;41(2): 327-32.

22 Inker LA, Schmid CH, Tighiouart H, Eckfeldt JH, Feldman HI, Greene T, et al. Estimating glomerular filtration rate from serum creatinine and cystatin C. N Engl J Med. 2012;367(1):20-9.

23 Hoffman JI, Buckberg GD. The myocardial oxygen supply:demand index revisited. J Am Heart Assoc. 2014; 3(1):e000285. 